\title{
Usability Testing of an E- Learning System: A Comparative study of two Evaluation Techniques
}

\author{
Sidra Shahid ${ }^{1}$, Muhammad Shabbir Abbasi ${ }^{2}$ \\ Isidrashahid@uoswomenfsd.edu.pk, ${ }^{2}$ abbasi_shabbir@yahoo.com \\ ${ }^{1,2}$ (Department of Computer Science \& IT, University of Sargodha Women Campus, Faisalabad, Pakistan)
}

\begin{abstract}
E- Learning is the most feasible way to hold distance learning by providing learning materials over the internet; thus trend is becoming popular in developed and developing countries. This learning style becomes difficult to use by learners, usually by vague interface design. Due to which learner/user becomes reluctant towards E- Learning system. Usability testing of E- Learning systems targets its interface design, usability and human computer interaction. This study is an attempt to uncover the problems that occur in the use of such systems at user end. A survey from learners of an E-Learning system is carried out and result are compared by another usability evaluation technique named Heuristic Evaluation commonly employed by experts. Both end users and usability expert participated in this research by which two different evaluation methods are used to evaluate the usability of Virtual University Learning Management System. At the end some suggestions are given in order to improve the usability of target system after validating the results.
\end{abstract}

Keywords: E- Learning, HCI (Human Computer Interaction), Usability Evaluation, Usability Evaluation Criteria.

\section{Introduction}

Internet has approached almost every corner of the world and has turned it into a global village. It has shown us a new dimension in order to remain connected with all sorts of information and facility. World Wide Web is an important pillar of internet consisting of ten million of websites and web user applications spread all over the world. It has induced too much extent into one's social to personal life. The facilities like E- Learning, E- Commerce, and E-government are just one click away, either on our desktop computers or mobile devices [1]. HCI is a necessary element in the success of computer science and has proved a broader and diverse field than computer science itself. More the element of quality interaction, more the success of the system of a product will be there. As Human Computer Interaction (HCI) is moving towards its new advanced directions the requirement is to recognize its importance [2]. Technologies and computers are becoming invisible with more power but giving a more intense feel of facilitation to society. To assess Human Computer Interaction and its value first consider its concept:

"Human Computer Interaction (HCI) is a discipline concerned with the design, evaluation and implementation of interactive computer systems for human use and with the study of major phenomena surrounding them", (Working definition in the ACM SIGCHI Curricula). HCI is emerged to make our work more natural with the help of technology even without noticing the presence of computers in our surroundings. Therefore multiple HCI directions and devices are rising [2]. The focal point of human computer interaction is area of usability. As usability is a tool or way of measuring and evaluating human computer interaction of a particular system or product. The slogan of this concept is basically "simple to be taught, simple to perform" [2]. Usability is mainly applied as evaluation and design rule. Usability by Jacob Neilson is defined as "usability is a standard of quality that evaluates the easiness of user interfaces. Usability is also defined as the way or technique for inducing user friendliness in the software during its design [3]. According to Neilson, Usability of comprised of five factors: Learnability, Efficiency, Memorability, Errors and Satisfaction.

Nielson's heuristics can be define as: field of vision associated with process rank, go with concerning process and also real life, individual command and also liberty, reliability and also specifications, problem reduction, acknowledgement as opposed to recollect, mobility and also productivity people, cosmetic and also minimalist pattern, help users understand, identify and also cure errors and also help and also records. As the technology is getting faster, more of the interactive user interfaces are being developed to give user a feel of real world out of a virtual world. More attention is being paid to interfaces by many researchers. As discussed above, there are many areas that have electronic shape such as E-Commerce, E- Learning, E-Shopping, E Banking, ELibrary, etc., thus this revolution was nothing without usability and usefulness [3]. There are various means to evaluate usability associated with an E- Learning application, including field testing, heuristic evaluations, observations, user check lists and usability testing. The heuristic evaluation can be a guideline as well as a standard. The heuristic evaluation can be a method that will involves the official application review by professionals with usability to check interface style in order to assess whether or not the system is designed with 
renowned principles with proven requirements of aesthetic user interfaces. True aim of heuristic evaluation is always to recognize prospective functionality along with convenience difficulties to be able to solve them just before final execution [4].

Usability test are performed by users on specific tasks in a controlled laboratory environment. The concluded metrics includes the level of success of user while performing a task, time taken by the user to finish a task and the satisfaction level that user achieved. Whereas field studies involve user performance while accomplishing the specific tasks in their own environments. The main advantage of field study is that they mitigate the affect of making assumption about user's style of application use. Moreover, filed studies provide insight view of the user's needs and wants. However, there are many challenge associated with usability evaluation of E- Learning applications. For instance, an E- Learning application must comply with diverse backgrounds and experiences of users [5].

What is the meaning of learning? Some explanations seem to only cope with activities [6], some only with details [7] and some cope with details and visible activities What all these explanations have in typical is that they all perspective studying as an improvement over time and that is designed at enhancing abilities to knowledge, through a sequence of activities through individual statement and connections [8]. These scientists also find another typical ground: in that they believe the fact studying is not a procedure of transferring details from someone who knows to someone who does not. Instead studying is an effective procedure that happens through immediate encounter, by being involved in genuine projects [8].

The major purpose for online learning is that it should be educationally valuable, it is essential in such surroundings to comprehend how functionality leads to (or not) to academic objectives [9]. [10] claimed that students have not considered the effects of usability functions of an academic program to experience academic objectives. Both writers suggest that there is a need to help test candidates consider the way in which functionality and studying communicate.

The main objective of this research is to perform usability testing of an E- Learning system to unveil the problems and criteria for interface design that need most attention while designing such systems in order to make them more usable. Another focal point of the study is comparison of two usability testing techniques to find which one is better in context of E- Learning system evaluation. In the same way this study will address that to which extent does the Heuristic Evaluation and survey results corresponds in a way to surface the usability problems of E- Learning System.

\section{Literature Review}

A research performed by the usability company [11] mentioned that useless interfaces will cost ELearning organizations enormous amounts simply because users will not be able to use their techniques effectively. The United States E- Learning industry has a estimated value of \$11.5 billion dollars by 2003 [12], while the Western industry is predicted to be worth $\$ 4$ billion dollars by this year (Frontend, 2001), however inadequate usability will give increase to frustrating results for many E- Learning organizations. There are several reasons for this, one of which is that E- Learning organizations are not including significant user groups: in The united states alone $8 \%$ of the inhabitants has visible learning, intellectual, hearing or physical skill problems to impact their access the Internet [11]. In Western countries the ratio is $11 \%$ and is predicted to increase to $18 \%$ by 2020 [11].

[13] expressed that Usability testing seems to be hardly ever used in the particular development involving E- Learning in professional medical education. The focus involving excellent examining may be the consumer of the specific item and it notifies item development with a methodical procedure to recognize difficulties from an early point throughout item development so that these kinds of difficulties can be usually rectified.

[14] researched that several researchers suppose that the robust partnership is out there in between excellent in addition to functionality, but this particular premises has not been experimentally assessed. This research reveals a robust likeness in between E- Learning quality and to E-Learning usability. When the functionality of E-Learning technique just isn't adequate, the particular fundamental functionality metrics should be improved. This involves a good analytical method to manage the particular metrics efficiently. But all is not improved due to the restrictions like time and money.

[15] stated that Usability tests inside the E- Learning area are certainly not very frequent. In spite of the significant position which usability represents inside the good results of each and every E- Learning process. The user interface should not only transparent in addition to easy to use, your learners/students target Elearning features rather than in buying articles. In addition, it is believed that usability reviews require further thought on the understanding viewpoint. Specifically, strategies to E- Learning usability cover anything to help E- Learning. Apparently, a possible recognized set of heuristics along with a joint proper evaluation for ELearning applications is not applied yet. This shows that there is certainly need of further research in addition to empirical evaluation. 
[5] stated that in traditional learning E- Learning techniques have got conquer the particular temporal and spatial restrictions involving traditional finding out situations inside extending educational opportunities to be able to everyone. Which has a development price involving 35.6\%, the particular E- Learning market can be increasing with an incredible speed, even now many E- Learning techniques are unsuccessful.

\section{Methodology}

VULMS (Virtual University Learning Management System) is one of the top ranked E- Learing system in Pakistan. It is widely used by learners as it provideshigh quality education by distance learning. It offers a variety of academic graduate and post graduate programs. The Virtual University of Pakistan delivers education through a judicious combination of broadcast television and the Internet. VU courses are hand-crafted in meticulous detail by acknowledged experts in the field. Lectures are then recorded in a professional studio environment and after insertion of slides, movie clips and other material, become ready for broadcast. Course lectures are broadcast over free-to-air television and are also made available in the form of multimedia CDs. They can also be made available as streaming media from the Virtual University's servers. The multiple formats allows for a high degree of flexibility for students who may view the lectures at a time of their choosing within a 24-hour period. Additionally, students can use the lectures to review an entire course before their examinations - a facility simply not available in the conventional face-to-face environment.

In addition to the prescribed texts, comprehensive reading material / lecture notes in the form of webenabled content are provided through a comprehensive Learning Management System (LMS) hosted on the VU Web Servers and accessible over the Internet. The full power of hyperlinks is utilized for making the on-line experience a truly powerful one. The LMS also provides an e-mail facility to each and every student as well as discussion boards for interaction within the VU community. An important feature of the LMS is a Question/Answer board where VU faculty provides answers to questions posed by students on the subject matter covered in the lectures. The Question/Answer board (also known as the Moderated Discussion Board) is extremely fine grained, providing separate sessions for each lecture of each course. In addition, read-only access is made available to previous question/answer sessions and this constitutes an extremely useful study resource for students. VU faculty monitors this board on a continuous basis and answers to student questions are provided within a short space of time.

First of all a deep literature review is performed to understand all the concepts related to this research. Then the attributes about E- Learning evaluation are filtered. This research is based on two stages. In first phase a questionnaire survey is conducted from VULMS users. In other phase Heuristic Evaluation is applied to get the verified results. Over all mixed research methodologies is adopted.

Questionnaires were planned with purpose of obtaining quantitative data. An objective for questionnaires was to find out strength and weakness of the systems. An online questionnare was disigned so that the users can find it over internet and respond instantly. Students of VU from different programs were selected but common thing was that they all use the system at same level. Questionnaire was consists of 25 questions from which 3 questions were open ended whereas 22 questions were close ended. 3 open ended questions were made to get user's opinions about system and user's demographic informations. Overall questionnaire was easy to understand.

Nielsen ten heuristics are used as Heuristic Evaluation Criteria. Neilsen's ten heuristics that are used for system's conformance are listed as under:

1. Visibility of System's Status

2. Match between system and real world

3. User control and freedom

4. Consistency and standards

5. Error prevention

6. Recognition rather than recall

7. Flexibility and efficiency of use

8. Aesthetics and minimalist design

9. Help user recognize, disgnose ad recover from errors

10. Help and documentation

To conduct heuristic evaluation, the first step is the selection of a usability expert. As usability in an evolving field in Pakistan that's why we could approach only one usability expert. Who has done much research work in the field of usability and human computer interaction. As he is a usability expert so we did not need much to explain to her about our intent of study. Overall HE was performed according to Nielsen's ten Heuristics and identified over all 28 interface problems in E- Learning System. The details of those identified problems are given below: 


\section{Visibility of System status and content:}

According to the results almost $40 \%$ of the respondents' votes for agree options for visibility of system status and contents. Whereas $30 \%$ voted for strongly agreed and almost $30 \%$ voted for the category of disagree to this feature. There were 4 questions in this category.

\section{Site Navigation, Organization and Structure:}

Three questions were asked for Site Navigation, Organization and Structure category. In which $40 \%$ respondents choose for strongly disagree and agree options respectively. Rest $30 \%$ of the respondents went for disagree and $30 \%$ for strongly agree options.

\section{Consistency and Relevance:}

For Consistency and Relevance 3 questions were asked. About $60 \%$ users voted for disagree options and rest $40 \%$ gone for agree options. Mostly people voted for low standard of this option.

\section{Learnability and Accessibility:}

The Learnability and Flexibility portion gives the following analytical results: about $60 \%$ of the users were agreed with this portion. Whereas rest $40 \%$ rated it below average.

\section{Efficiency and flexibility:}

In this $5^{\text {th }}$ portion of questionnaire three questions were asked. In which above $50 \%$ voted for disagree options to show that they are not satisfied with this aspect of system. Rest of the people voted for agree options.

\section{Help and Support:}

In the $6^{\text {th }}$ portion of questionnaire again three questions were asked. About $75 \%$ users respond towards agree options and $25 \%$ respond towards disagree options.

\section{Effectiveness and Satisfaction:}

In this section mostly student consider the system below satisfaction as about 55\% respondents voted for disagree options whereas rest $45 \%$ users responded for agree options.

\section{Result And Discussions}

Different techniques and methods are used worldwide to assess the usability. Each usability assessment method/technique variations different mind techniques. It is not necessary that all the usability assessment techniques give same outcomes. Assume, if one specific strategy resolves all the problems regarding usability, then there is no need for any others strategy. In order to get more improve outcomes, questionnaire and heuristic evaluation are used as main device to assess the system.

According to [16], task in an information-rich globe is not only to create details available to individuals whenever and wherever they need and in any type but particularly to say the right factor at the perfect period of time in the right way. Particularly referring to the pedagogy sector where student goals are reliant on the details offered by program, analyzing functionality is of excellent significance

The results and findings of both the methods are similar up to much extent. The following are the criteria obtained by the combination of both the techniques. That criterion is grouped into main heading like Visibility, Feedback, Tolerance, Consistency, Simplicity and Structure.

\section{Conclusion}

Main objective of this research was not only the assessment of the usability of user interface of an ELearning application but also to evaluate the interface support to learner's studying actions. Usability assessment is done empirically and different techniques were chosen for this purpose. One usability assessment techniques could not recognize all usability problems so multi-method strategy has been implemented by including those users who have regular connections with the system. Different usability assessment techniques can provide different results because each technique variations different mind techniques. Some techniques are more accurate and contain actual theoretical information. On the other hand, some give mathematical information which enable evaluator to evaluate result.

In pedagogy sector usability has significant significance and used as primary parameter to assess the usability of E- Learning technology. Studying style effects on learning, so good user interface means accomplishing higher user fulfillment level. Two major methods were used in this usability assessment of VULMS interface: Heuristic Evaluation and survey. Discussions were performed to confirm the research of results. For any E- Learning application, evaluating requirements for success is that it should integrate with the three primary features of usability i.e. efficiency, effectiveness and user satisfaction. This research determines 
that after development as recommended in suggestions by this research, E- Learning could be more efficient and effective for users.

\section{References}

[1]. U. Yousf, L. Khaw, H. Ch'ng and B.J. Neow, Balancing between usability and aesthetics of Web design, Information Technology (ITSim) 2010 International Symposium in U.K., 1(1), 2010, 1-6.

[2]. D. Sharma, Toward a new foundation of human-computer interaction, Electronics Computer Technology (ICECT), 2011 3rd International Conference, 6(1), 2011, 395-397.

[3]. J, Nielsen, and R.L. Mack, Usability Inspection Methods (John Wiley and Sons, Inc., 1994).

[4]. A. Hope, and P. Guiton, Strategies for Sustainable Open and Distance E- Learning, Routledge Taylor and Francis Group, Madison Ave., New York. 2006.

[5]. D. Dhar, and P. Yammiyavar, Design Approach for E- Learning Systems: Should it be User Centered or Learner Centered. Technology for Education (T4E), IEEE Fourth International Conference, 5(1), 2012, 239-240.

[6]. R. Schunk, Rapid prototyping of patterned functional nanostructures, Nature international weekly Journal of Science. 2010, 56-60.

[7]. M.D. Myers, Qualitative Research in Information Systems ( London, 1997).

[8]. P. Zaharias, K. Vassilopoulou, and A. Poulymenakou, Designing On-line Learning Courses: Implications for Usability, Scientific Journal on Applied Information Technology 1 (1), 2002, 1-12.

[9]. A. Jones, E. Scanlon, C. Tosunoglu, E. Morris , S. Ross, P. Butcher, and J. Greenberg, Contexts for evaluating educational software. Interacting with Computers, 11(1), 1999, 499-516.

[10]. D. Squires, and J. Preece, Predicting quality in educational software: Evaluating for learning, usability and the synergy between them. Interacting with Computers, 11 (5), 1999, 567-483.

[11]. FrontEnd.com. ,2001. Why people can't use eLearning: What the eLearning industry needs to learn about usability. 1-16.

[12]. C.R. Abernathy, Magnetic and structural properties of Mn-implanted GaN, Applied Physics Letters. 78(22), 2001, 3475 - 3477

[13]. J. Sandars, and N. Lafferty, Twelve Tips on usability testing to develop effective E Learning in medical education, Med Teach, 32(12), 2010, 956-60.

[14]. A.Oztekin, Z. Kong and O. Uysal, UseLearn: A novel checklist and usability evaluation method for eLearning systems by criticality metric analysis. International Journal of Industrial Ergonomics, 40(1), 2010, 455-469.

[15]. A.Granića, and M. Ćukušić, Usability Testing and Expert Inspections Complemented by Educational Evaluation: A Case Study of an E- Learning Platform, Educational Technology \& Society, 14 (2), 2011, 107-123.

[16]. D. Fallman, Design-oriented human-computer interaction, CHI '03 Proceedings of the SIGCHI Conference on Human Factors in Computing Systems, 2003, 225-232. 